\title{
28 Research Square \\ Effect of socioeconomic status on risk of COVID-19: a population-based case-control study
}

Hüseyin Küçükali ( $\square$ hkucukali@medipol.edu.tr)

Istanbul Medipol University, School of Medicine https://orcid.org/0000-0003-1669-3107

Osman Hayran

Istanbul Medipol University, School of Medicine

Şeyda İleri

Istanbul Medipol University, School of Medicine

\section{Berkay Kurt}

Istanbul Medipol University, School of Medicine

Fatmanur Kuru

Istanbul Medipol University, School of Medicine

Sena Nalbantoğlu

Istanbul Medipol University, School of Medicine

Burcu Özbaş

Istanbul Medipol University, School of Medicine

Mehmet Akif Sezerol

Health Directorate of Uskudar

Yusuf Taşçı

Health Directorate of Uskudar

\section{Research Article}

Keywords: COVID-19, SARS-CoV-2, Socioeconomic Status, Health Disparities

Posted Date: August 16th, 2021

DOI: https://doi.org/10.21203/rs.3.rs-195468/v2

License: (c) (1) This work is licensed under a Creative Commons Attribution 4.0 International License.

Read Full License 


\section{Abstract}

Most of the studies investigating the relationship between socioeconomic status and the occurrence of COVID-19 have limitations due to their ecological design. To investigate the relationship between socioeconomic status and individual-level risk of developing COVID-19, we conducted a populationbased, age and gender matched, case-control study in a district of Istanbul. The case group was defined as all confirmed COVID-19 cases $(n=232)$ in the district in a week of August 2020. The control group was matched with cases in terms of age and gender and selected randomly from the directory of the same primary care provider in the district in a 1:1 ratio. The socioeconomic status was measured by the Turkish Socioeconomic Status Index which considers its three dimensions: education, occupation, and income. 168 cases (72.4\% response) and 168 controls are included in the study. The mean socioeconomic status score is $70.28 \pm 7.09$ for cases and $69.25 \pm 7.46$ for controls $(p=0.201) .15 .2 \%(n=25)$ of cases and $22.4 \%$ $(n=37)$ of controls is in lower socioeconomic status group $(p=0.091)$. The analysis did not reveal a statistically significant difference between study groups. Socioeconomic status may not a significant predictor of COVID-19 occurrence at the individual-level. The disease seems to be a common threat to all individuals.

\section{Introduction}

In December 2019, a new respiratory system infectious disease thought to be first spread from a live animal market in Wuhan China, soon became a pandemic. The causative agent is defined as SARS-CoV-2 from the family of viruses known as coronaviruses. The disease, called COVID-19, initially started as smaller clusters in many countries. As of February 2020, COVID-19 cases increased rapidly in most of Europe, the United States, Australia, Asia, and Africa. World Health Organization declared a global pandemic on March 11, 2020. COVID-19 has affected 220 countries, caused 78,194,947 million cases and 1,736,752 million deaths by December 25th, 2020 [1] and continues to harm everyday life worldwide.

A better understanding of the factors that influence the risk of developing an infection, would benefit the planning of current health care services and provide necessary information to prevention or modelling studies for both current and future epidemics. Available statistical data show that higher age, male sex and pre-existing chronic diseases are significant risk factors for the negative effects of COVID-19, but the roles of socioeconomic factors are not fully understood yet.[2] Studies regarding the relationship between Socioeconomic Status (SES) and the occurrence of COVID-19 disease are usually ecological studies and have limitations due to ecological fallacy.[3-5] On the other hand, most individual-level studies are hospital-based and more focused on ethnic and racial disparities than SES.[6-8]

Socioeconomic factors are considered major determinants in life expectancy, well-being, and health. SES is a combination determined by variables such as education level, occupation, income level [9] and reveals inequalities between individuals towards privileges or resources.[10] 
It is well-known that low socioeconomic status increases all-cause morbidity and mortality.[11] Previous studies suggest a link between low socioeconomic level and increased risk of infectious diseases and impaired immune responses. People of low socioeconomic status should be considered high-risk populations in the event of an epidemic. Their status can affect the prognosis and severity of the disease also.[9]

Early research on the social epidemiology of COVID-19 indicates the impact of socioeconomic status on infection and deaths, as people of lower SES facing more harm.[12],[13] Although it is known that many diseases are more common among those who suffer from conditions such as poor housing conditions, overcrowded living, lack of financial security [12] it is difficult to say similar relationship for COVID-19 since the socioeconomic characteristics of COVID-19 patients are not routinely recorded.

There are other risk factors better known than SES for COVID-19 transmission and disease development such as older ages, pre-existing chronic diseases (especially heart diseases, hypertension, diabetes, and chronic obstructive pulmonary disease) and smoking.[14] The increased ACE-2 enzyme, which also enables coronaviruses to enter the cells, is suspected reason in disease mechanism.[15] In diabetes, high blood sugar also damages the person's immune system and the low immune system is more susceptible to infections.[16] Smoking is also known for increasing the risk of developing a variety of viral infections and their complications.[17] To understand the effect of SES on COVID-19 infection, it is necessary to control the confounding effects of such known factors.

It is difficult to determine the risk of developing infections between different social groups. One of the main problems here is that information about ethnicity and socioeconomic status is not available adequately in routine health data. Besides, the sizes of different social groups in the population are not completely known.[2] However, to effectively prevent further spread with appropriate interventions, identify people who are more prone to develop disease or become severely ill and to better treat them; it is critical to understand socioeconomic factors at every dimension of the epidemic.[9] Epidemiologic studies on the issue would be useful for identifying the most vulnerable groups and planning more equitable public health interventions. For this reason, the aim of this study is to identify the effect of socioeconomic status on the risk of developing COVID-19.

\section{Materials And Methods}

To identify the effect of socioeconomic status on the risk of developing COVID-19, we conducted a population-based, age and gender matched, case-control study in September 2020 in the Uskudar district of Istanbul, Turkey. With more than five hundred thousand dwellers, Uskudar is the eighth-most populated district among thirty-nine districts in Istanbul. COVID-19 incidence in the district was similar to other districts at the time of the study.

Assuming 44.4\% prevalence of lower socioeconomic status among the population [18] and anticipated 1.93 risk ratio for developing COVID-19 [19], the minimum required sample size was calculated as 320 for $80 \%$ power, $95 \%$ confidence level, and 1:1 case-control ratio. 
To minimize the risk of selection bias, we adopted a population-based approach in participant recruitment. Turkish national public health information system (known as HSYS) reports any COVID-19 case that is identified by any public or private laboratory to the district health directorate (local administrative body of the Ministry of Health) of their residence. Based on this system, we defined the case group as all laboratory-confirmed COVID-19 cases identified between August 19th and 25th and reside in Uskudar district $(n=232)$ and recruited via the District Health Directorate of Uskudar. $168(72.4 \%)$ of them accepted to participate in the study.

We defined the control group as randomly selected healthy people who were residing in Uskudar and were matched with cases in terms of age, gender and primary healthcare provider (i.e. family physician) for eliminating the possible confounding effects. Controls were selected from the primary healthcare registry of the District Health Directorate of Uskudar. According to the primary health care system in Turkey, all citizens had been assigned to a public primary healthcare provider (i.e. family physician) based on their residence by default. This registry consisted of 538.293 people. For each case in the study, the control is randomly selected from the list of people of the same age, gender, and primary healthcare provider in this registry. Because primary healthcare provider information is not available for 50 cases, their controls could not match in terms of primary healthcare providers.

Random selection of controls was done via software developed by a researcher for this specific purpose in the Python programming language. Participants who were not living currently in Uskudar and the controls who had current or previous laboratory-confirmed COVID-19 diagnoses were excluded from the study. Controls were resampled until all cases were matched with a control.

Potential participants were approached via telephone calls, informed, and included in the study if they give consent. When they could not be reached, they were called again up to 3 consecutive days at different hours.

Researchers entered data into a structured and online data collection form. Collected data consisted of age, gender, size of household, education levels of households, occupations of households, total net monthly income of the household, pre-existing chronic disease, and amount of smoking (pack-year).

Socioeconomic status score (SES score) was measured by the Turkish Socioeconomic Status Index (TÜSES) and an online calculator (available on http://tyap.net/ses) developed by Social Structure Studies Program research group.[18] This scale considers three dimensions of socioeconomic status: education, occupational prestige, and income. The scale uses the highest educational level and occupational prestige among households and the mean monthly income per household. Occupational prestige has been operationalized with a score that represents the prestige of 133 occupation categories in the society as is indicated in a preliminary study. Distinctly from many other alternatives, this scale is based on households, and its validity and reliability are controlled in the Turkish population.

A previous study on a representative sample from Turkey, defined $44.4 \%$ of the population as lower SES group, following $38.8 \%$ as lower-middle, following $14.6 \%$ as upper-middle and last $2.2 \%$ as upper SES 
group. SES score thresholds for grouping have been defined as $65.26,70.95$, and 78 respectively.[18]

Some participants hesitated to give information about their income, and some others do not know the exact amount of income of other households. To overcome this issue, we followed the guidance of Galobardes \& Demarest [20] while asking a question about income. We first asked the exact total amount of all households' incomes. In the case that participant hesitates to answer we asked if it is higher or lower than the minimum wage. Following their answer, we prompted income intervals. After detecting the interval, we tried to narrow the interval until the participant feels fine. Before data analysis, income intervals transformed into an average value of its lower and upper boundaries. Yet three cases have not provided income data at all and hence a SES score could not be calculated for them. These 3 pairs are excluded from income-related comparisons only.

Data were analyzed in SPSS v22 software. Continuous variables (age, years of completed education, smoking pack-year, household size, occupational prestige score, monthly income per household, and SES score) were summarized with mean and standard deviation for both study groups. Counts and percentages are given for other variables. Differences between study groups are tested by t-test for continuous and chi-square test for categorical variables. To identify the change in risk of developing COVID-19 odds ratio is calculated for categorical variables. Conditional logistic regression was conducted to identify the influencing factors on the odds of developing COVID-19. The statistical significance threshold was considered as 0.05 .

This study is approved by the Ministry of Health General Directorate of Healthcare on 25.08.2020 and by the Ethics Committee of Istanbul Medipol University on 03.09.2020 (No: 664). All methods were carried out following relevant guidelines and regulations.

\section{Results}

A total of 168 cases ( $72.4 \%$ response rate) and 168 controls are included in the study. Demographic characteristics of participants by study groups are given in Table 1. Because cases and controls are matched by age and gender there is no difference between the age and gender distribution of the groups and $48.8 \%$ of participants are female and $51.2 \%$ are male and the mean age is $37.66 \pm 15.32$ for each of the study groups.

Table 1. Demographic characteristics of participants by study groups 


\begin{tabular}{lllll} 
& Cases & \multicolumn{3}{c}{ Controls } \\
\hline & Mean & S.D. & Mean & S.D. \\
\hline Age $^{\text {a }}$ & 37.66 & 15.32 & 37.66 & 15.32 \\
\hline & $\mathbf{n}$ & $\%$ & $\mathbf{n}$ & $\%$ \\
\hline Gender $^{\text {a }}$ & & & & \\
\hline Male & 86 & 51.2 & 86 & 51.2 \\
\hline Female & 82 & 48.8 & 82 & 48.8 \\
\hline Education ${ }^{b}$ & & & & \\
\hline Illiterate & 11 & 6.5 & 11 & 6.5 \\
\hline Primary school & 24 & 14.3 & 28 & 16.7 \\
\hline Secondary school & 84 & 50.0 & 65 & 38.7 \\
\hline University & 49 & 29.2 & 64 & 38.1 \\
\hline Total & 168 & 100.0 & 168 & 100.0
\end{tabular}

\footnotetext{
a Matching variable.

b Comparison between groups: $\chi 2=4.722 p=0.193$
}

The percentage of university graduates is higher among controls than cases $(38.1 \%$ and $29.2 \%$ respectively) however there is no statistically significant difference between the educational level of case and control groups.

Table 2 summarizes the distribution of participants' pre-existing chronic diseases by study groups. The percentage of people who have any pre-existing chronic diseases in cases $(20.8 \%)$ is not significantly different than in controls $(20.2 \%)(p=0.893)$.

Table 2. Distribution of participants' pre-existing chronic diseases by study groups 


\begin{tabular}{lllllll} 
& \multicolumn{7}{l}{ Cases } & \multicolumn{2}{l}{ Controls } & & \\
\cline { 2 - 7 } & $\mathbf{n}$ & $\%$ & $\mathbf{n}$ & $\%$ & $\chi^{2 \mathbf{b}}$ & $\mathbf{p}$ \\
\hline Allergy & 0 & 0 & 2 & 1.2 & $\mathrm{n} / \mathrm{a}$ & - \\
\hline Asthma or COPD & 8 & 4.8 & 1 & 0.6 & $\mathrm{n} / \mathrm{a}$ & - \\
\hline Cancer & 1 & 0.6 & 0 & 0 & $\mathrm{n} / \mathrm{a}$ & - \\
\hline Diabetes & 8 & 4.8 & 12 & 7.1 & 0.85 & 0.35 \\
\hline Heart diseases & 6 & 3.6 & 3 & 1.8 & $\mathrm{n} / \mathrm{a}$ & - \\
\hline Hepatitis & 1 & 0.6 & 1 & 0.6 & $\mathrm{n} / \mathrm{a}$ & - \\
\hline Hypertension & 17 & 10.1 & 15 & 8.9 & 0.13 & 0.71 \\
\hline Thyroid diseases & 4 & 2.4 & 0 & 0 & $\mathrm{n} / \mathrm{a}$ & - \\
\hline Rheumatic diseases & 1 & 0.6 & 3 & 1.8 & $\mathrm{n} / \mathrm{a}$ & - \\
\hline Kidney diseases & 0 & 0 & 4 & 2.4 & $\mathrm{n} / \mathrm{a}$ & - \\
\hline Other & 1 & 0.6 & 3 & 1.8 & $\mathrm{n} / \mathrm{a}$ & - \\
\hline One or more ${ }^{a}$ & 35 & 20.8 & 34 & 20.2 & 0.02 & 0.89 \\
\hline None & 133 & 79.2 & 134 & 79.8 & & \\
\hline Total & 168 & 100 & 168 & 100 & & \\
\hline
\end{tabular}

a Since participants may have more than one disease, the sum of counts of individual diseases are not equal to count for "One or more".

${ }^{b}$ Comparisons are in-between disease's presence and absence. For variables other than Diabetes, Hypertension and "One or more" groups, $\chi^{2}$ tests are not applicable because $50 \%$ of expected values are smaller than five.

Mean smoking pack-year was $2.52 \pm 7.93$ for cases and $3.73 \pm 8.23$ for controls, and the difference between groups is not statistically significant $(t=-1.379 p=0.169)$.

The distribution of variables that contribute to the socioeconomic status by study groups is summarized in Table 3. Although cases have slightly lower educational years, occupational prestige scores and slightly higher household size, monthly income per household and socioeconomic status score than controls; no statistically significant difference can be detected for any of those variables between the two groups.

Table 3. Components of the socioeconomic status by study groups 


\begin{tabular}{lllllll} 
& \multicolumn{7}{c}{ Cases } & \multicolumn{2}{c}{ Controls } \\
\cline { 2 - 7 } & Mean & S.D. & Mean & S.D. & t & p \\
\hline Educational years & 10.15 & 4.34 & 10.38 & 4.48 & 0.384 & 0.701 \\
\hline Household size & 3.65 & 1.65 & 3.55 & 1.32 & 0.062 & 0.536 \\
\hline Occupational prestige score & 32.26 & 29.86 & 34.43 & 29.49 & 0.671 & 0.503 \\
\hline Monthly income per household $^{\text {a }}$ & $6,659.61$ & $5,710.34$ & $5,550.00$ & $6,421.92$ & 1.659 & 0.098 \\
\hline Socioeconomic status score $^{\mathrm{a}}$ & 70.28 & 7.09 & 69.25 & 7.46 & 1.280 & 0.201
\end{tabular}

a These calculations are based on 165 pairs of cases and controls. Others are based on 168 pairs.

Table 4, presents the categorical SES data of the study groups. As it is seen from Table 4, the distribution of cases and controls by socioeconomic status groups which are formed by thresholds defined in the study of Sunar \& Kaya [18], does not reveal any significant difference in terms of developing COVID-19.

Table 4. Distribution of participants by socioeconomic status groups and study groups

\begin{tabular}{|llllllll|}
\hline \multicolumn{7}{l}{ Cases } & \multicolumn{2}{l}{ Controls } & & & \\
& $\mathbf{n}$ & $\%$ & $\mathbf{n}$ & $\%$ & $\chi^{2 \mathbf{a}}$ & $\mathbf{p}^{\mathbf{a}}$ & OR $^{\mathbf{a}}(95 \% \mathbf{C l})$ \\
\hline Lower & 25 & 15.2 & 37 & 22.4 & 2.860 & 0.091 & $0.62(0.35-1.08)$ \\
\hline Lower-Middle & 78 & 47.3 & 71 & 43.0 & 0.600 & 0.439 & $1.19(0.77-1.83)$ \\
\hline Upper-Middle & 44 & 26.7 & 39 & 23.6 & 0.402 & 0.526 & $1.18(0.71-1.93)$ \\
\hline Upper & 18 & 10.9 & 18 & 10.9 & 0.000 & 1.000 & $1.00(0.50-2.00)$ \\
\hline Total & 165 & 100.0 & 165 & 100.0 & & & \\
\hline
\end{tabular}

a Each group in comparison with all other groups combined.

We analyzed the study data by logistic regression to understand the probable predictors for the occurrence of COVID-19 cases. Results of logistic regression analysis are presented in Table 5. As it is seen from the table none of the factors of interest seems to be a significant predictor of being a COVID-19 case.

Table 5. Predictors of being a COVID-19 case by logistic regression analysis 


\begin{tabular}{|llllllll|}
\hline Independent variables & $\mathbf{B}$ & $\mathbf{S E}$ & Wald & $\mathbf{p}$ & $\boldsymbol{\beta}$ & $\mathbf{9 5 \%} \mathbf{C l}$ \\
\cline { 1 - 4 } & & & & & & Lower & Upper \\
\hline Pre-existing chronic disease & -0.005 & 0.340 & $<0.001$ & 0.988 & 0.995 & 0.511 & 1.938 \\
\hline Smoking (pack-year) & -0.020 & 0.016 & 1.708 & 0.191 & 0.980 & 0.950 & 1.010 \\
\hline Education years & -0.032 & 0.041 & 0.593 & 0.441 & 0.969 & 0.894 & 1.050 \\
\hline Household size & 0.075 & 0.085 & 0.776 & 0.378 & 1.078 & 0.913 & 1.273 \\
\hline Occupational prestige score & -0.006 & 0.006 & 1.104 & 0.293 & 0.994 & 0.982 & 1.006 \\
\hline Monthly income per household & $<0.001$ & $<0.001$ & 0.013 & 0.908 & 1.000 & 1.000 & 1.000 \\
\hline Socioeconomic status score & 0.037 & 0.031 & 1.366 & 0.243 & 1.037 & 0.975 & 1.103 \\
\hline
\end{tabular}

Dependent variable: 0-Control, 1-Case

\section{Discussion}

As it was stated at the introduction of this paper several studies have reported a close relationship between the frequency of COVID-19 cases and old age, pre-existing chronic conditions and smoking habits, but the roles of socioeconomic factors are not fully understood yet.[2]

According to our findings of a population-based case-control study, there is no statistically significant difference between COVID-19 cases and matched controls with regard to the pre-existing chronic conditions and smoking habits of the individuals. The distribution of the pre-existing chronic diseases and frequency of smoking are similar for both case and control groups. It is known that pre-existing chronic diseases influence the prognosis of COVID-19 and contributes to high fatality rates. Because their relationship with the occurrence of the COVID-19 is not clear, our finding regarding the pre-existing chronic conditions is reasonable.

Another important finding of our study is the lack of a significant difference between SES levels of the cases and controls. This finding was against our expectation and also not compatible with other study findings which indicate associations between neighborhoods with a large dependent youth population, densely populated, low-income, and predominantly colored neighborhoods, and COVID-19 test positivity rate. $[5,6,21,22]$ However, most of these studies have ecological design and comparisons are not done at the individual level. We conclude that the population-based, matched case-control and individual-level data collection structure of our study make our findings trustworthy.

The association of SES with several health problems including infectious diseases has a long history and it is the source of the discipline Social Epidemiology.[23] SES is a composite index and a multidimensional measure determined by variables such as education level, occupation, income level. It is an important variable during the investigation of public health problems, however, there are 
measurement difficulties due to its complex nature. In this study, we used a scale that has high validity and reliability which makes our findings meaningful. This scale takes all the three essential dimensions of socioeconomic status into consideration: education, occupational prestige, and income.

Education has influences on Socioeconomic Status in many ways. As the education level increases, the level of social and health awareness of the individuals and household increases. Within the scope of an infectious disease outbreak, individuals with limited health literacy which is associated with a lower level of education may overlook the measures to be taken to contain the epidemic and may be misled by misinformation. The effectiveness of public health communication during the pandemic also depends on people's ability to access and understand messages. Furthermore, lower levels of education can be indirectly associated with many factors that may suppress the immune system such as increased smoking and malnutrition.[9] Most of the professions with higher status and income can be attained through education.

Occupations of people may put them at risk due to the nature of the work. An occupation that requires constant face-to-face interaction with people may ease the spreading of or receiving an infection through droplets. Moreover, people in some occupations are more likely to suffer work-related stress, burnout, job insecurity and unemployment that all can lead to impaired immune and inflammatory system responses. $[9,11]$ It is shown that severe COVID-19 cases are more likely to be workers and less likely to be people working in their own work.[9]

The income level of the person or household is the major determining factor for nutrition, housing, and health expenditures. The low-income level can cause crowded households, more deprived neighborhoods, and poor housing conditions. It is known that these households are subjected to increased risk of transmission of many pathogens such as tuberculosis bacillus, Helicobacter Pylori or Ebstein-Barr virus. $[2,9]$ Impoverished population groups have difficulties in adopting preventive measures, such as social isolation, and if infection occurs, they have limited access to health services.[4] For a family struggling in poverty, a new economic recession due to the pandemic and measures to cover it can worsen physical and social conditions, thereby making them even more vulnerable to the impact of COVID-19.[9]

As a result, although several study findings support the well-known public health cliché "People with low SES are under high risk of infectious diseases", we did not find any significant SES difference between COVID-19 cases and their age and gender matched controls.

However, this result should be concluded carefully since our study has some limitations. The most important limitation of our study is its cross-sectional nature. Longitudinal study designs, especially, follow-up studies are needed to investigate the causal relationship. However, it was not feasible to organize and conduct a follow-up or cohort study under pandemic conditions. Another limitation is the size of our sample. Our sample size is representative for investigating the effect of SES but not sufficient for making subgroup conclusions for all the "pre-existing conditions". Besides these limitations, the population-based design of this study makes the results more valuable and meaningful. Because both 
cases and controls are obtained from the population and not from admissions of specific hospitals or clinics, it is reasonable to assume this study is free from most of the selection biases.

\section{Conclusions}

Our findings indicate neither protective nor worsening effect of socioeconomic status on risk of developing COVID-19. The disease should be seen as a common threat to society. As Burström and Tao have stated, scientific studies of inequalities in Coronavirus disease 2019 (COVID-19) are lacking at present, but it is reasonable to assume that disparities in social determinants of health have contributed to some early observations and result in differential exposure to the virus, differential vulnerability to the infection and differential consequences of the disease.[24] We urge that population-based follow-up studies should be conducted to generate reliable evidence about the effects of SES on COVID-19 occurrence.

\section{Declarations}

Author Contributions: Conceptualization, $\mathrm{HK}, \mathrm{OH}$; methodology, $\mathrm{HK}, \mathrm{OH}$; software, $\mathrm{HK}$; formal analysis, $\mathrm{HK}$, Şi, BK, FK, SN, BÖ; investigation, HK, Şi, BK, FK, SN, BÖ; resources, OH, HK, MAS, YT; data curation, HK, Şi, BK, FK, SN, BÖ, MAS, YT; writing-original draft preparation, HK, OH, Şi, BK, FK, SN, BÖ; writing-review and editing, $\mathrm{HK}, \mathrm{OH}$, ŞI, BK, FK, SN, BÖ, MAS, YT; visualization, HK; supervision, $\mathrm{HK}, \mathrm{OH}$; project administration HK. All authors have read and agreed to the published version of the manuscript.

Funding: This research received no external funding.

Institutional Review Board Statement: The study was conducted according to the guidelines of the Declaration of Helsinki and approved by the Ethics Committee of Istanbul Medipol University (Date: 03.09.2020 No: 664) after the administrative approval of the Ministry of Health General Directorate of Healthcare (Date: 25.08.2020).

Informed Consent Statement: Informed consent was obtained from all subjects involved in the study. Due to safety restrictions regarding the ongoing pandemic, participants were approachable only via telephone calls. Therefore, the consent of participants is obtained verbally through telephone calls. Interviewers first read the information script which explains the study's aim and method, rights of participants, and data privacy. Interviewers asked study questions only to those who clearly give consent.

Data Availability Statement: The dataset used and analyzed during the current study are available from the corresponding author on reasonable request.

Acknowledgments: The authors would like to thank the administrative staff of the District Health Directorate of Uskudar for their assistance in approaching participants.

Conflicts of Interest: The authors declare no conflict of interest. 


\section{References}

1. World Health Organization. WHO Coronavirus Disease (COVID-19) Dashboard. Available online: https://covid19.who.int/ (accessed on 2 December 2020).

2. Niedzwiedz, C.L.; O'Donnell, C.A.; Jani, B.D.; Demou, E.; Ho, F.K.; Celis-Morales, C.; Nicholl, B.I.; Mair, F.S.; Welsh, P.; Sattar, N.; et al. Ethnic and socioeconomic differences in SARS-CoV-2 infection: prospective cohort study using UK Biobank. BMC Med. 2020, 18, 160, doi:10.1186/s12916-020-01640-8.

3. Figueroa, J.F.; Wadhera, R.K.; Mehtsun, W.T.; Riley, K.; Phelan, J.; Jha, A.K. Association of race, ethnicity, and community-level factors with COVID-19 cases and deaths across U.S. counties. Healthc. (Amst) 2020, 9, 100495, doi:10.1016/j.hjdsi.2020.100495.

4. Souza, C.D.F. de; Machado, M.F.; do Carmo, R.F. Human development, social vulnerability and COVID-19 in Brazil: a study of the social determinants of health. Infect. Dis. Poverty 2020, 9, 124, doi:10.1186/s40249-020-00743-x.

5. Whittle, R.S.; Diaz-Artiles, A. An ecological study of socioeconomic predictors in detection of COVID19 cases across neighborhoods in New York City. BMC Med. 2020, 18, 271, doi:10.1186/s12916-02001731-6.

6. Lieberman-Cribbin, W.; Tuminello, S.; Flores, R.M.; Taioli, E. Disparities in COVID-19 Testing and Positivity in New York City. Am. J. Prev. Med. 2020, 59, 326-332, doi:10.1016/j.amepre.2020.06.005.

7. Price-Haywood, E.G.; Burton, J.; Fort, D.; Seoane, L. Hospitalization and Mortality among Black Patients and White Patients with Covid-19. N. Engl. J. Med. 2020, 382, 2534-2543, doi:10.1056/NEJMsa2011686.

8. Richardson, S.; Hirsch, J.S.; Narasimhan, M.; Crawford, J.M.; McGinn, T.; Davidson, K.W.; Barnaby, D.P.; Becker, L.B.; Chelico, J.D.; Cohen, S.L.; et al. Presenting Characteristics, Comorbidities, and Outcomes Among 5700 Patients Hospitalized With COVID-19 in the New York City Area. JAMA 2020, 323, 20522059, doi:10.1001/jama.2020.6775.

9. Khalatbari-Soltani, S.; Cumming, R.C.; Delpierre, C.; Kelly-Irving, M. Importance of collecting data on socioeconomic determinants from the early stage of the COVID-19 outbreak onwards. J. Epidemiol. Community Health 2020, 74, 620-623, doi:10.1136/jech-2020-214297.

10. Holuka, C.; Merz, M.P.; Fernandes, S.B.; Charalambous, E.G.; Seal, S.V.; Grova, N.; Turner, J.D. The COVID-19 Pandemic: Does Our Early Life Environment, Life Trajectory and Socioeconomic Status Determine Disease Susceptibility and Severity? Int. J. Mol. Sci. 2020, 21, doi:10.3390/ijms21145094.

11. Mattos Dos Santos, R. Isolation, social stress, low socioeconomic status and its relationship to immune response in Covid-19 pandemic context. Brain Behav. Immun. Health 2020, 7, 100103, doi:10.1016/j.bbih.2020.100103. 
12. Mikolai, J.; Keenan, K.; Kulu, H. Intersecting household level health and socio-economic vulnerabilities and the COVID-19 crisis: An analysis from the UK. SSM Popul. Health 2020, 100628, doi:10.1016/j.ssmph.2020.100628.

13. Kaiser, J.C.; Stathopoulos, G.T. Socioeconomic correlates of the SARS-CoV 2 and Influenza H1N1 outbreaks. Eur. Respir. J. 2020, doi:10.1183/13993003.01400-2020.

14. Leung, J.M.; Niikura, M.; Yang, C.W.T.; Sin, D.D. COVID-19 and COPD. Eur. Respir. J. 2020, 56, doi:10.1183/13993003.02108-2020.

15. Guan, W.-J.; Ni, Z.-Y.; Hu, Y.; Liang, W.-H.; Ou, C.-Q.; He, J.-X.; Liu, L.; Shan, H.; Lei, C.-L.; Hui, D.S.C.; et al. Clinical Characteristics of Coronavirus Disease 2019 in China. N. Engl. J. Med. 2020, 382, 1708-1720, doi:10.1056/NEJMoa2002032.

16. Haybar, H.; Kazemnia, K.; Rahim, F. Underlying Chronic Disease and COVID-19 Infection: A State-ofthe-Art Review. Jundishapur J Chronic Dis Care 2020, In Press, doi:10.5812/jjcdc.103452.

17. Bauer, C.M.; Morissette, M.C.; Stämpfli, M.R. The Influence of Cigarette Smoking on Viral Infections. Chest 2013, 143, 196-206, doi:10.1378/chest.12-0930.

18. Sunar, L.; Kaya, Y.; Otrar, Y. Türkiye sosyoekonomik statü endeksi geliştirme projesi. (TÜBITAK: 113K506). Available online: https://app.trdizin.gov.tr/publication/project/detail/TVRVMk16WTI.

19. Prats-Uribe, A.; Paredes, R.; Prieto-Alhambra, D. Ethnicity, comorbidity, socioeconomic status, and their associations with COVID-19 infection in England: a cohort analysis of UK Biobank data, 2020.

20. Galobardes, B.; Demarest, S. Asking sensitive information: an example with income. Soz. Praventivmed. 2003, 48, 70-72, doi:10.1007/s000380300008.

21. van Dorn, A.; Cooney, R.E.; Sabin, M.L. COVID-19 exacerbating inequalities in the US. The Lancet 2020, 395, 1243-1244, doi:10.1016/S0140-6736(20)30893-X.

22. Wang, Z.; Tang, K. Combating COVID-19: health equity matters. Nat. Med. 2020, 26, 458, doi:10.1038/s41591-020-0823-6.

23. Berkman, L.F.; Kawachi, I. Social epidemiology; Oxford University Press: Oxford, 2000, ISBN 0195083318.

24. Burström, B.; Tao, W. Social determinants of health and inequalities in COVID-19. Eur. J. Public Health 2020, 30, 617-618, doi:10.1093/eurpub/ckaa095.

\section{Supplementary Files}


This is a list of supplementary files associated with this preprint. Click to download.

- GraphicalAbstract.png 